\title{
Hypoxic Preconditioning Augments Efficacy of Human Endothelial Progenitor Cells for Therapeutic Neovascularization
}

\author{
Takako Akita, Toyoaki Murohara, Hisao Ikeda, Ken-ichiro Sasaki, \\ Toshifumi Shimada, Kimiyasu Egami, and Tsutomu Imaizumi
}

The Cardiovascular Research Institute and Department of Internal Medicine III (TA, TM, HI, K-iS, TS, KE, TI), Kurume University School of Medicine, Kurume, Department of Cardiology (TM), Nagoya University Graduate School of Medicine, Nagoya, Japan

SUMMARY: A subset of human peripheral blood mononuclear cells (PB-MNCs) differentiate into endothelial progenitor cells (EPCs) that participate in postnatal neovascularization. Although tissue ischemia can mobilize EPCs from bone marrow, the effects of hypoxia on differentiation and angiogenic function of EPCs are little known. We examined whether hypoxic conditioning would modulate differentiation and function of human PB-MNC-derived EPCs. A subset of PB-MNCs gave rise to EPC-like attaching (AT) cells under either normoxic or hypoxic conditions. However, hypoxia much enhanced the differentiation of AT cells from PB-MNCs compared with normoxia. AT cells released vascular endothelial growth factor (VEGF) protein and expressed CD31 and kinase insert domain receptor/VEGFR-2, endothelial lineage markers, on their surface, which were also enhanced by hypoxia. Both a neutralizing anti-VEGF mAb and a KDR-specific receptor tyrosine kinase inhibitor, SU1498, suppressed PB-MNC differentiation into EPC-like AT cells in a dose-dependent manner. Migration of AT cells in response to VEGF as examined by a modified Boyden chamber apparatus was also enhanced by hypoxia. Finally, in vivo neovascularization efficacy was significantly enhanced by in vitro hypoxic conditioning of AT cells when cells were transplanted into the ischemic hindlimb of immunodeficient nude rats. In conclusion, hypoxia directly stimulated differentiation of EPC-like AT cells from human PB-MNC culture. Moreover, hypoxic preconditioning of AT cells before in vivo transplantation is a useful means to enhance therapeutic vasculogenesis. (Lab Invest 2003, 83:65-73).

$A$ ngiogenesis is defined as the formation of new blood vessels by sprouting of preexisting mature endothelial cells (ECs) (Carmeliet, 2000; Folkman, 1995; Risau, 1997). In contrast, vasculogenesis is referred to as the creation of primordial blood vessels from endothelial progenitor cells (EPCs) or angioblasts (Risau, 1995, 1997). Neovascular formation in adults has been considered to result exclusively from the former process (ie, angiogenesis). However, we and other researchers recently identified EPCs in adult human peripheral blood, and EPCs have been shown to accumulate at active angiogenic foci and to participate in neovascularization, a notion consistent with "postnatal vasculogenesis" (Asahara et al, 1997, 1999; Lin et al, 2000; Murohara et al, 2000; Vasa et al, 2001).

\section{DOI: 10.1097/01.LAB.0000050761.67879.E4}

Received August 21, 2002.

Supported by grants from the Ministry of Education, Science, and Culture, the Ministry of Health and Welfare of Japan, the Kimura Foundation, and the Japan Heart Foundation.

Address reprint requests to: Dr. Toyoaki Murohara, The Cardiovascular Research Institute, Kurume University, Department of Cardiology, Nagoya University Graduate School of Medicine, 65 Tsurumai, Showa-Ku, Nagoya, 466-8550, Japan.E-mail: murohara@med.nagoya-u.ac.jp
EPCs in adults are believed to mobilize from bone marrow (BM) (Asahara et al, 1999; Gunsilius et al, 2000; Takahashi et al, 1999), but EPCs can also differentiate from peripheral blood mononuclear cells (PB-MNCs) during culture in vitro (Asahara et al, 1997). The latter finding suggests that EPCs first leave BM, and then more mature EPCs may differentiate in situ at periphery in response to local angiogenic stimuli. One can hypothesize that a subset of circulating PB-MNCs home to vessels located in active angiogenic foci (eg, ischemic tissues), and these cells may differentiate into EC lineage cells in situ.

Although tissue ischemia (hypoxia) is well known to mobilize EPCs from BM (Shintani et al, 2001a; Takahashi et al, 1999), it is little known as to whether differentiation of EPCs from PB-MNCs can be stimulated by local hypoxic condition and whether angiogenic functions of EPCs are enhanced by hypoxia. Accordingly, we examined whether hypoxia itself would influence the differentiation of human PB-MNCs into EPCs during culture and whether angiogenic properties of EPCs would be enhanced under hypoxic conditions. We also explored potential mechanism(s) by which differentiation and functions of EPCs are modulated by hypoxic conditioning. 


\section{Results}

\section{Differentiation of Adult Human PB-MNCs into EPC-Like Attaching Cells During Culture}

Under either normoxic or hypoxic conditions, human PB-MNCs formed a number of cell clusters during culture in the presence of EC growth supplements on gelatin-coated plastic plates. EPC-like attaching (AT) cells then differentiated and sprouted from the cell clusters. AT cells showed limited ( $<5 \%$ cells) expression of CD45, a common leukocyte antigen, but expressed CD31 and kinase insert domain receptor (KDR) (Fig. 1A), endothelial-specific lineage markers. Furthermore, more than $70 \%$ of AT cells on Day 7 of culture took up Dil-acLDL (Fig. 1A). Thus, AT cells acquired multiple EC-like characteristics, and thus we defined the AT cells as a major population of EPCs (EPC-like AT cells).

\section{Hypoxia Augmented the EPC Differentiation from Human PB-MNCs}

When culture plates were exposed to hypoxia for 7 days, more cell clusters and EPC-like AT cells developed from hypoxic PB-MNCs than from normoxic PB-MNCs (Fig. 1B). On both Day 5 and day 7 of culture, the numbers of cell clusters and EPC-like AT cells were 1.5 -fold $(p<0.001)$ and 2 -fold $(p<0.001)$ greater in the hypoxia group than in the normoxia group, respectively (Fig. 1C).

\section{Hypoxia Augmented the Expression of CD31 and KDR/VEGFR-2 on EPC-Like AT Cells}

We next examined the expression of EC lineage markers on EPC-like AT cells using flow cytometry. Immediately after isolation of PB-MNCs, only a small number of PB-MNCs expressed KDR $(<1 \%)$ and CD31 $(<5 \%)$, respectively. On Day 7 of culture, the percentage of KDR- or CD31-positive AT cells was significantly increased and was greater in the hypoxia group than in the normoxia group ( $p<0.05$, hypoxia versus normoxia) (Fig. 2).

\section{Hypoxia Augmented the VEGF Release from PB-MNCs}

Immunocytochemical examination revealed that EPCs expressed vascular endothelial growth factor (VEGF) protein in both normoxia-conditioned EPCs and hypoxia-conditioned EPCs (Fig. 3A). Intracellularly produced VEGF can be secreted from intact cells because VEGF has a hydrophobic leader sequence on its amino terminus (Leung et al, 1989). We therefore examined the effects of hypoxia on the VEGF produc-

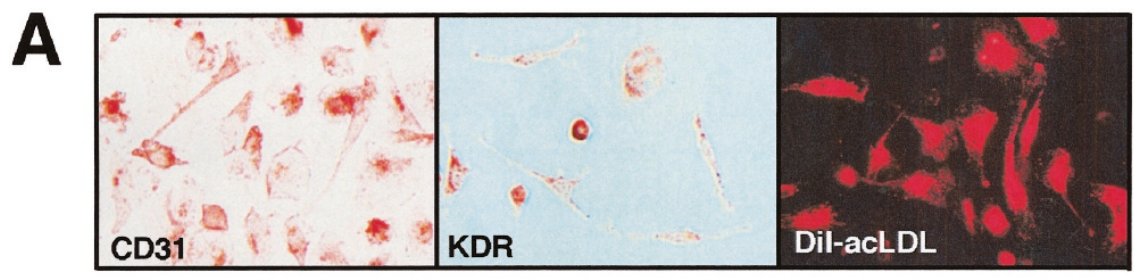

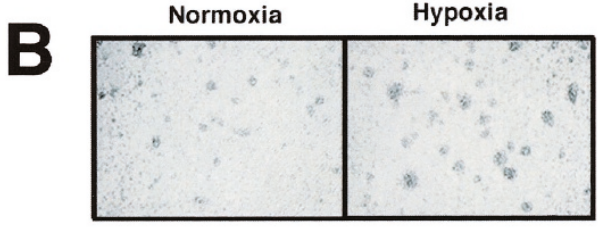

Cell Clusters
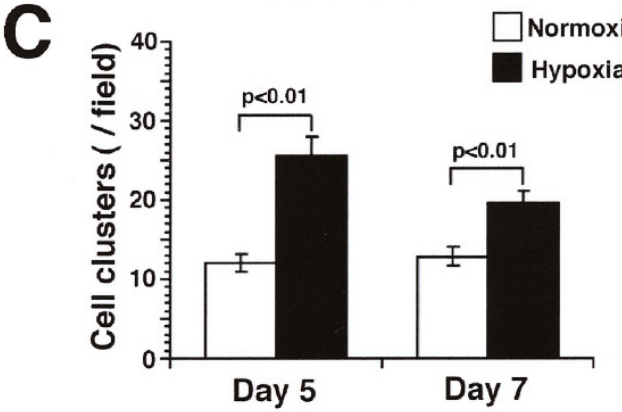



Attaching Cells



Figure 1.

A, Endothelial progenitor cell (EPC)-like attaching (AT) cells expressed CD31 and KDR/NEGFR-2 (EC lineage markers) and incorporated Dil-labeled acetylated low-density lymphocytes (LDL). Panels show representative photomicrographs of normoxia-conditioned EPC-like AT cells. B, Greater number of cell clusters and EPC-like AT cells differentiated from peripheral blood mononuclear cells (PB-MNCs) cultured in the hypoxic condition than from those cultured in the normoxic condition. C, Quantitative analysis shows that on Day 7 of culture the number of cell clusters and EPC-like AT cells was significantly greater in the hypoxia group than in the normoxia group. 
A

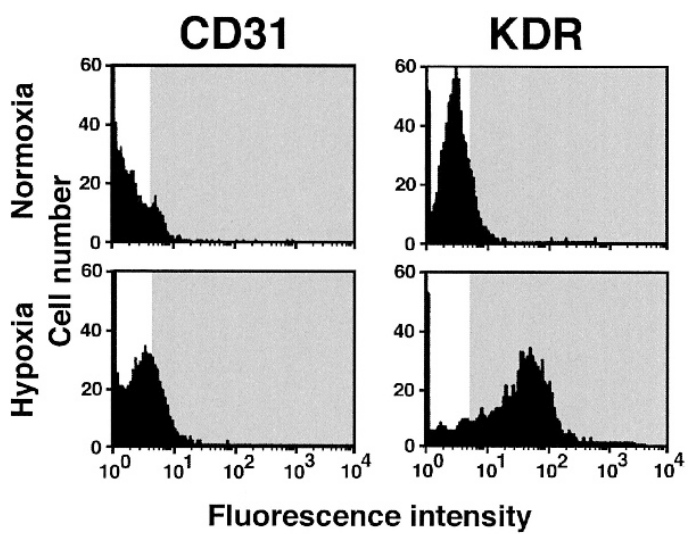

B

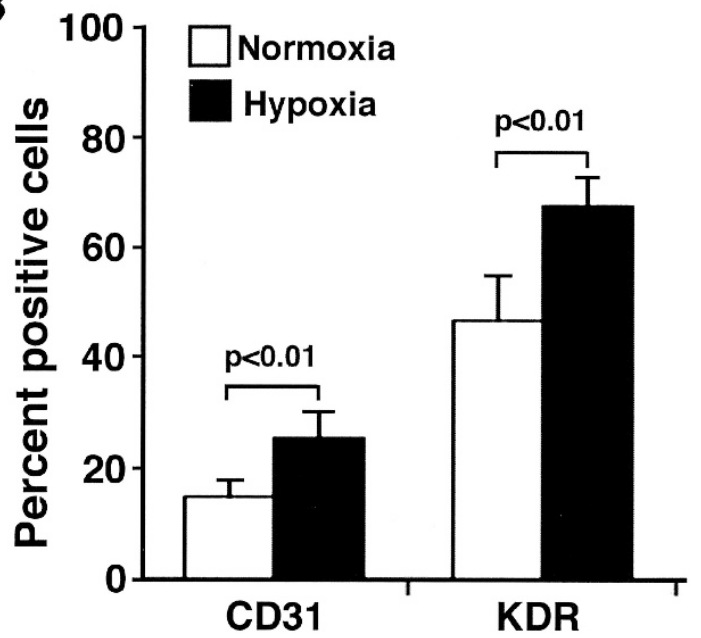

Figure 2.

A, Hypoxia augmented expression of CD31 and KDR (EC lineage markers) on EPC-like AT cells as assessed by flow cytometry. B, On Day 7 of culture, the percentage of KDR- or CD31-positive EPC-like AT cells was significantly greater in the hypoxia group than in the normoxia group.

tion from cultured human PB-MNCs. The concentration of VEGF in culture media on Day 7 was significantly greater in the hypoxia group than in the normoxia group (308 \pm 90 versus $253 \pm 79 \mathrm{pg} / \mathrm{mL}, p$ $<0.05)$ (Fig. 3B). Thus, hypoxia significantly augmented VEGF production and release from PB-MNCs in culture during EPC differentiation.

\section{VEGF Plays an Important Autocrine Role in the Differentiation of Human PB-MNCs into EPCs}

We examined the effects of exogenous rhVEGF protein $(10 \mathrm{ng} / \mathrm{mL})$ on the differentiation of PB-MNCs into EPCs. VEGF significantly enhanced the differentiation of PB-MNCs into EPCs in vitro (Fig. 4A). To elucidate the role of autocrine VEGF released from $\mathrm{PB}-\mathrm{MNCs}$ on their self differentiation into EPC-like AT cells, we examined the effects of the neutralizing anti-VEGF $\mathrm{mAb}$, the tyrosine kinase inhibitor genistein, and the KDR-specific receptor tyrosine kinase inhibitor, SU1498, on differentiation of EPCs from PB-MNC. All of these agents potently inhibited the differentiation of PB-MNCs into EPCs as judged by the number of
A

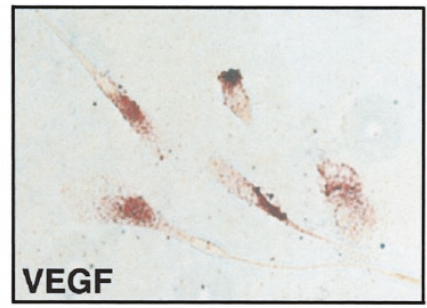

B

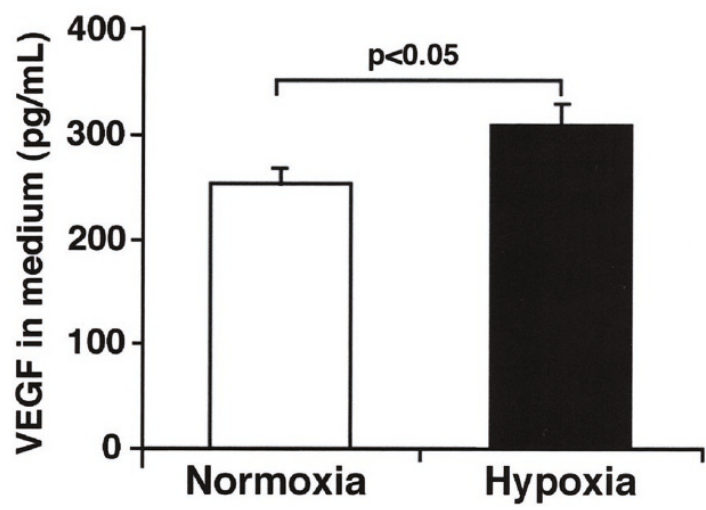

Figure 3

A, Immunocytochemical examination revealed that EPC-like AT cells expressed VEGF protein intensively. The panel shows a representative photomicrograph taken from normoxia-conditioned EPC-like AT cells. B, VEGF concentration in culture medium on Day 7 was significantly greater in the hypoxia group than in the normoxia group.

differentiated EPC-like AT cells. Importantly, the neutralizing anti-VEGF mAb and SU1498 significantly suppressed the differentiation of AT cells in a dosedependent manner (Fig. 4).

\section{Hypoxia Enhanced Migratory Function of EPCs in Response to VEGF}

We examined whether migration of EPCs, an essential early step in the process of vasculogenesis, is enhanced under the hypoxic condition. Boydenchamber experiments revealed that a greater number of hypoxia-conditioned EPCs migrated in response to rhVEGF (10 ng/mL) compared with normoxiaconditioned EPCs (Fig. 5).

\section{Cell Transplantation-Mediated Neovascularization is Enhanced by Hypoxic Preconditioning of EPC-Like AT Cells before In Vivo Transplantation}

Finally, we examined whether hypoxia-preconditioned ECP-like AT cells had a greater efficacy on in vivo neovascularization compared with normoxiaconditioned AT cells by employing a nude-rat model of unilateral hindlimb ischemia. Green fluorescencelabeled transplanted EPCs participated in capillary formation in the ischemic hindlimb tissues (Fig. 6A). Next, serial laser Doppler blood flow (LDBF) analyses revealed that the ischemic/normal hindlimb LDBF ratio was significantly greater in both the hypoxiaconditioned EPC and normoxia-conditioned EPC transplantation groups as compared with the saline- 

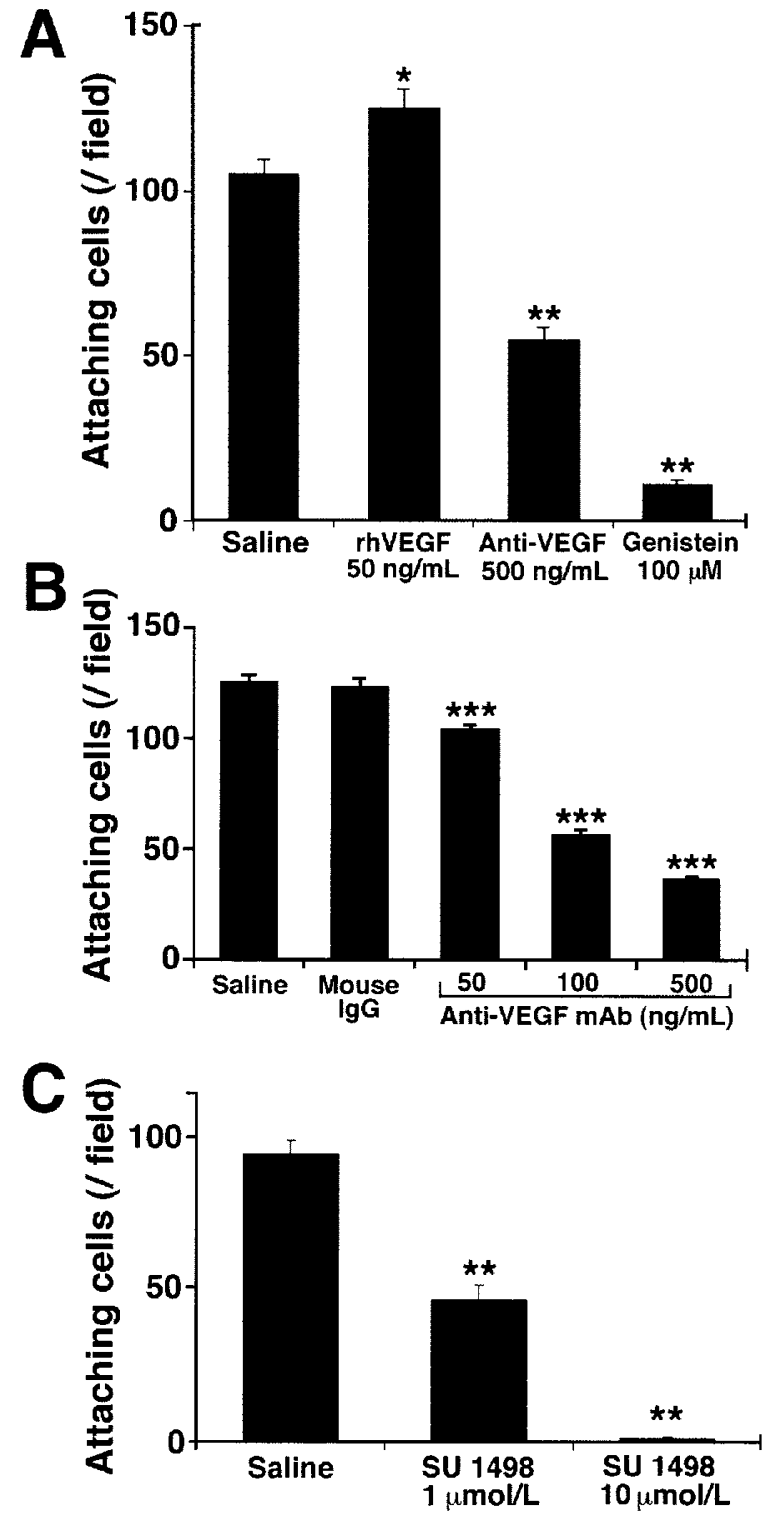

Figure 4.

A, Exogenous addition of rhVEGF (10 $\mathrm{ng} / \mathrm{mL})$ significantly enhanced the differentiation of PB-MNCs into EPC-like AT cells. The neutralizing anti-VEGF $\mathrm{mAb}$ and the tyrosine kinase inhibitor genistein significantly inhibited the differentiation of PB-MNCs into EPCs. B, The neutralizing anti-VEGF mAb inhibited the differentiation of PB-MNCs into EPC-like AT cells in a dosedependent manner. But this effect was not observed in the group of cells treated with the highest concentration of a control nonimmune IgG. C, The KDR-specific tyrosine kinase inhibitor SU1498 significantly inhibited the differentiation of PB-MNCs into EPCs in a dose-dependent manner. ${ }^{*} p<0.05$, ${ }^{* \star} p<0.01,{ }^{* \star *} p<0.001$ versus the saline group.

injected control group (Fig. 6, B and C). Moreover, the recovery of the ischemic/normal hindlimb LDBF ratio was greater in the hypoxia-conditioned EPCtransplanted group than in the normoxia-conditioned EPC-transplanted group. Thus, (a) transplantation of EPC-like AT cells functionally contributed to therapeutic neovascularization in ischemic tissues in vivo, and (b) the efficacy for neovascularization was significantly enhanced by hypoxic preconditioning of EPCs before transplantation.
A

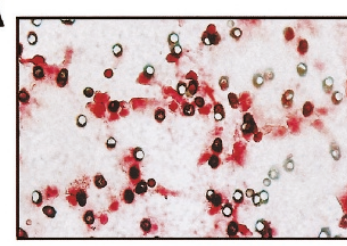

Normoxia

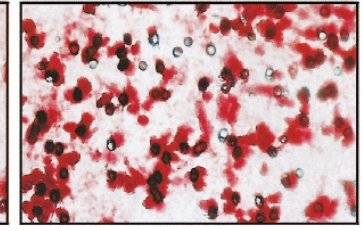

Hypoxia

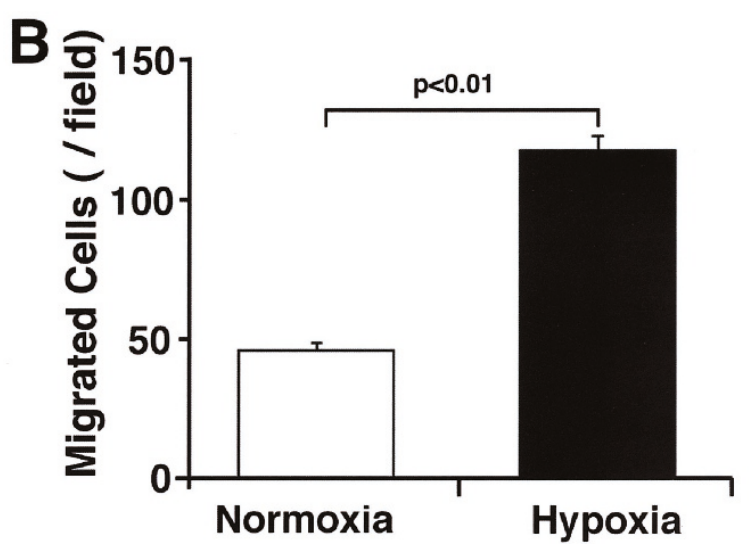

Figure 5.

A, Greater number of hypoxia-conditioned EPC-like AT cells migrated in response to VEGF (10 ng/mL) compared with normoxia-conditioned EPC-like AT cells. Migrated cells attached on the lower surface of transwell membrane are stained brown. B, A greater number of EPC-like AT cells migrated in response to VEGF when they were cultured in the hypoxic condition than in the normoxic conditions.

\section{Discussion}

The present study demonstrated that hypoxia directly enhanced the differentiation of human PB-MNCs into EPCs in vitro, and that EPCs cultured under hypoxic conditions exhibited greater angiogenic properties. Namely, EPCs exposed to hypoxic conditions showed an enhanced migratory activity in vitro and greater angiogenic efficacy after they were transplanted into the ischemic hindlimb of immunodeficient nude rats.

We previously showed that a subset of human PB-MNCs differentiated into EPCs during culture and that the number of circulating EPCs increased after acute tissue ischemia in humans (Shintani et al, 2001a). However, the direct effects of hypoxia on differentiation of PB-MNCs into EPCs and their angiogenic properties were little known. In the present study, the number of EPCs derived from a fixed amount of human PB-MNC culture (5000 cells $\left./ \mathrm{mm}^{2}\right)$ was significantly larger in the hypoxia group than in the normoxia group. Moreover, a greater number of hypoxia-conditioned EPCs expressed EC-lineage markers, CD31 and KDR, as compared with normoxiaconditioned EPCs. These results suggest that hypoxia enhanced the differentiation of human PB-MNCs into EPCs during culture for 7 days.

We next examined the potential mechanisms by which hypoxia enhanced PB-MNC differentiation into EPCs. MNCs and ECs have been shown to produce and release VEGF, a potent angiogenic cytokine (Hojo et al, 2000; Namiki et al, 1995). In the present study, 
A

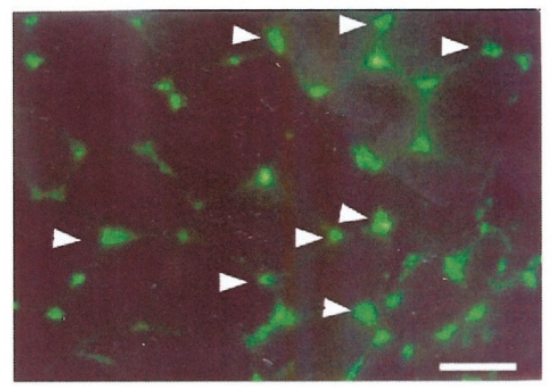

B

B Saline

EPC-TX

EPC-Tx

Before
surgery

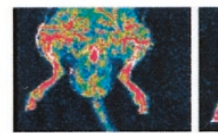

normoxia

hypoxia

After
surgery

Day 7
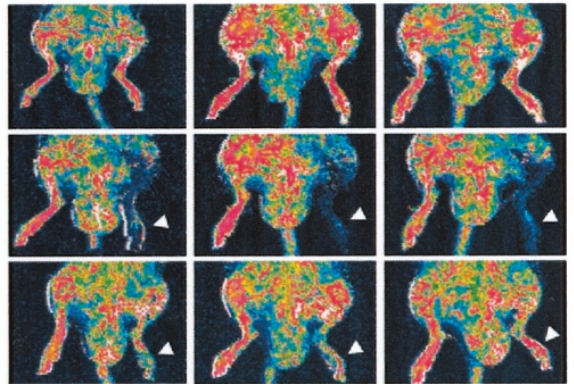

Day 14
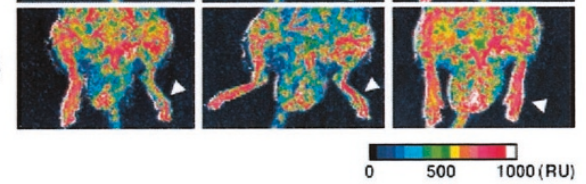

\section{C}

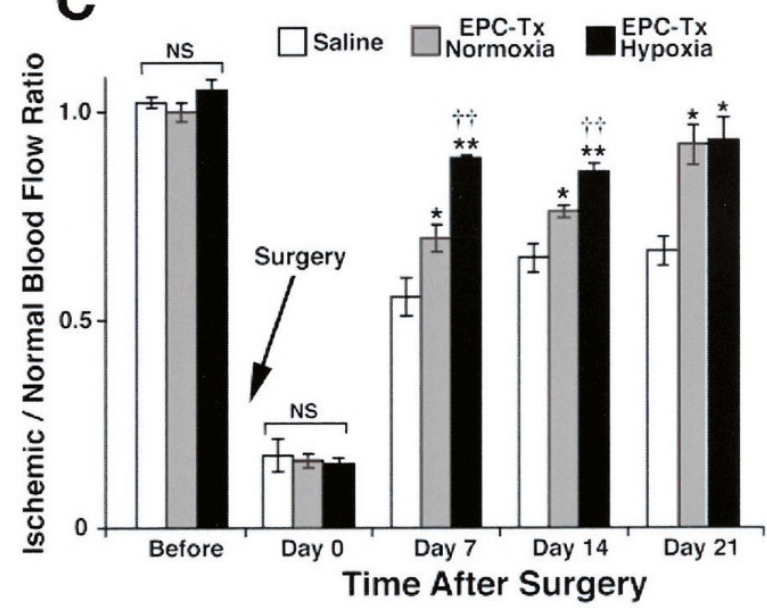

Figure 6.

A, Green fluorescence-labeled transplanted EPCs participated in capillary formation in the ischemic hindlimb tissues. Each green fluorescence signal represents EPC-derived capillaries with lumen structure (white arrows); bar = $50 \mu \mathrm{m}$. B, The ischemic hindlimb blood flow recovered readily in the two EPC-like AT cell transplanted (Tx) groups (normoxia and hypoxia EPC-Tx groups) compared with the saline-treated control group. The recovery of the ischemic hindlimb blood flow was greater in the hypoxia-conditioned EPC group than in the normoxia-conditioned EPC group. Panel C. Hindlimb blood flow was quantitatively analyzed by the ischemic/normal hindlimb blood flow ratio. The recovery of the laser Doppler blood flow (LDBF) ratio was significantly greater in the hypoxia-conditioned EPC-transplanted group than in the normoxia-conditioned EPC-transplanted group. The ratios in two EPCtransplantation groups are still greater than those in the control saline-treated group. ${ }^{*} p<0.05,{ }^{*} p<0.01$ versus the saline group, $\dagger^{\star}+p<0.01$ the normoxia versus hypoxia group.

immunocytochemical staining revealed that PB-MNCs and EPCs expressed VEGF protein. The intracellularly produced VEGF can be secreted from intact cells (Leung et al, 1989). On Day 7 of culture, the VEGF concentration in culture medium of EPCs was significantly higher in the hypoxia group than in the normoxia group. This finding is in agreement with previous reports showing that the VEGF gene promoter contains a hypoxia-responsive element, and that hypoxia enhances VEGF gene expression and VEGF protein secretion (Shweiki et al, 1992). Because VEGF is an important molecule for the differentiation and maturation of ECs (Millauer et al, 1993), the VEGF secreted from PB-MNCs during culture may play an important role in differentiation of PB-MNCs into EPCs by an autocrine mechanism. Consistently exogenous VEGF administration significantly enhanced the differentiation of PB-MNCs into EPCs in the present study. Thus, hypoxia-induced VEGF secretion may at least in part account for the enhanced differentiation of PBMNCs into EPCs under hypoxia.

To further clarify the above issue, we tested the effects of the neutralizing anti-VEGF $m A b$ and tyrosine kinase inhibitors on differentiation of PB-MNCs into EPCs. VEGF elicits the angiogenic function in ECs mainly through the receptor tyrosine kinase KDR/ Flk-1. That is, VEGF induces EC proliferation and increases vascular permeability mainly via the KDR/ Flk-1 (Kanno et al, 2000; Millauer et al, 1993; Murohara et al, 1998b). In the present study, the neutralizing anti-VEGF mAb, the tyrosine kinase inhibitor genistein, and the KDR-specific tyrosine kinase inhibitor SU1498 all inhibited the differentiation of PB-MNCs into EPCs. In particular, the neutralizing anti-VEGF $m A b$ and SU1498 suppressed the PB-MNC differentiation into EPCs in a dose-dependent manner. These findings clearly suggest that the autocrine VEGF released from PB-MNCs, and the VEGF signaling through the KDR receptor tyrosine kinase on EPC play important roles in the differentiation of PB-MNCs into EPCs.

Another interesting finding of the present study was that hypoxia enhanced the angiogenic properties of EPCs. First, the migration of EPCs in response to VEGF was significantly greater in the hypoxiaconditioned EPC group than in the normoxiaconditioned EPC group. This finding is relevant because EC migration is an early essential step for the process of neovascularization (Carmeliet, 2000; Folkman, 1995). Second, and more importantly, hypoxiaconditioned EPCs elicited greater angiogenic properties than normoxia-conditioned EPCs after they were transplanted into the ischemic hindlimb of immunodeficient nude rats (ie, therapeutic vasculogenesis by EPC transplantation). Thus, hypoxia enhanced not only the differentiation of PB-MNCs into EPCs in vitro but also the angiogenic activities of EPCs in vivo.

There may be several possible explanations for the enhancement of the in vivo angiogenic properties of EPC-like AT cells by hypoxic preconditioning. First, the release of VEGF from EPCs might be greater in the hypoxia group than in the normoxia group. The VEGF gene promoter contains the hypoxia-responsive element that reacts with transcription factor hypoxia 
inducible factor (HIF)- $1 \alpha$, and VEGF transcription is enhanced. The augmented release of VEGF may at least in part account for the enhanced angiogenic efficacy with hypoxia-conditioned EPCs. Second, KDR and CD31 expression on EPC-like AT cells was enhanced by hypoxia. Because VEGF stimulates EC migration and angiogenesis mainly through KDR (Kanno et al, 2000; Millauer et al, 1993), the increased KDR expression observed under hypoxic condition may also account for the enhanced angiogenic properties of hypoxia-conditioned EPCs. Our data are consistent with those of a study by Brogi et al (1996) who reported that hypoxia stimulated KDR expression on ECs. Interestingly, a recent study showed that the VEGF-KDR signaling pathway also induces KDR expression as a positive feedback mechanism (Shen et al, 1998). Thus, augmented autocrine VEGF release might have enhanced the expression of KDR on EPCs (Brogi et al, 1996; Shen et al, 1998). Taken together, the enhancement of VEGF release and KDR expression on EPCs by hypoxic conditioning may explain the greater proangiogenic properties of hypoxia-conditioned EPCs.

\section{Clinical Implications}

Therapeutic angiogenesis is an effective strategy to rescue ischemic tissues in patients with critical limb ischemia (Isner and Asahara, 1999, Tateishi-Yuyama et al, 2002). We and other investigators recently reported that transplantation of culture-expanded human EPCs augmented ischemia-induced angiogenesis in vivo (Kalka et al, 2000; Murohara et al, 2000). Similarly, direct implantation of autologous BM-MNCs containing EPCs has been shown to augment angiogenesis in ischemic tissues (Shintani et al, 2001b, Tateishi-Yuyama et al, 2002). Thus, cell transplantation appears to be an effective means for therapeutic neovascularization. However, its angiogenic efficacy is sometimes limited because of a shortage of transplantable autologous EPCs or BM-MNCs. The present findings may provide one possible clue to overcoming this issue. Namely, in vitro hypoxic preconditioning of culture-expanded EPCs or BM-MNCs before in vivo transplantation may enhance the angiogenic efficacy of these cells, which may eventually compensate for the shortage of EPCs or BM cells.

In summary, hypoxia directly enhanced the differentiation of PB-MNCs into EPCs. Moreover, hypoxiaconditioned EPCs exhibited greater angiogenic properties compared with normoxia-conditioned EPCs. Our findings raise the new concept that hypoxia not only mobilizes EPCs from BM but also promotes in situ differentiation of PB-MNCs into EPCs within local ischemic tissues. Finally, in vitro hypoxic preconditioning of EPCs or BM-MNCs before transplantation may be a potentially useful strategy to increase the efficacy of cell-mediated therapeutic neovascularization (ie, cell therapy).

\section{Materials and Methods}

\section{Peripheral Blood Samples}

Peripheral venous blood samples (200 mL each) were obtained from 38 healthy male volunteers (mean age $=31$ ) who were taking no medications. Each sample was collected into a sterile pack containing citratedextrose solution as the anticoagulant (SC-200; Terumo, Tokyo, Japan), and was used within 6 hours. Informed consent was obtained from all subjects, and the protocol was approved by our institutional review board.

\section{Mononuclear Cell Isolation and Culture}

MNCs were isolated from peripheral blood by centrifugation through a Histopaque-1077 (Sigma, St. Louis, Missouri) density gradient (Asahara et al, 1997; Murohara et al, 2000). Cells were cultured in Medium-199 supplemented with $20 \%$ fetal bovine serum (FBS), bovine pituitary extract (as EC growth supplements), heparin $(100 \mu \mathrm{g} / \mathrm{mL}$ ), and antibiotics (Invitrogen, Carlsbad, California; basal EC medium). MNCs were cultured on gelatin-coated (Sigma) 6-well plastic plates at a density of 5000 cells $/ \mathrm{mm}^{2}$.

MNCs obtained from each donor were cultured in two 6-well plastic plates. One plate was placed in a standard incubator under $95 \%$ air and $5 \% \mathrm{CO}_{2}$ (normoxia group), and the other was placed in a hypoxic chamber. To expose cells to hypoxia, an anoxic gas mixture $\left(95 \% \mathrm{~N}_{2}\right.$ and $\left.5 \% \mathrm{CO}_{2}\right)$ was infused into the hypoxic chamber for 10 minutes at a rate of $3 \mathrm{~L} / \mathrm{min}$ (Brogi et al, 1996; Murohara et al, 1999). The chamber was then placed in an incubator maintained at $37^{\circ} \mathrm{C}$. The anoxic gas mixture was replaced everyday. The $\mathrm{pO}_{2}$ of the culture medium reached a nadir of $35 \mathrm{~mm}$ $\mathrm{Hg}$ after a single anoxic gas infusion, whereas the $\mathrm{pO}_{2}$ of the cell culture in the normoxic incubator was maintained at around $150 \mathrm{~mm} \mathrm{Hg}$ (Murohara et al, 1999).

\section{Identification of EPCs}

During culture of PB-MNCs under either normoxic or hypoxic conditions, numerous cell clusters as well as spindle-shaped and AT cells appeared. AT cells with endothelial features (EPC-like AT cells) were characterized by the incorporation of Dil-labeled acetylated LDL and Ulex lectin binding (Asahara et al, 1997). The cell clusters and EPC-like AT cells were counted under light microscopy on Day 7 of culture. Five randomly selected microscopic fields in each well were examined, and the mean number of cell clusters and EPC-like AT cells (per field) was calculated and compared between the normoxic and hypoxic groups.

\section{Flow Cytometric Analysis of Cell Surface Markers}

Using flow cytometry, EPC differentiation was evaluated on the basis of the expression of KDR (VEGFR-2) and CD31, two EC-specific markers (DeLisser et al, 1997; Garlanda et al, 1997; Yamaguchi et al, 1993). 
First, fresh PB-MNCs were subjected to flow cytometric analysis. Second, after PB-MNCs were cultured under either normoxic or hypoxic conditions for 7 days, the medium was removed, and AT cells were washed with PBS. The cells were incubated with PBS containing $1 \mathrm{mmol} / \mathrm{L}$ EDTA (pH 7.4) for 20 minutes at $37^{\circ} \mathrm{C}$, and the detached cells were collected into plastic tubes. After centrifugation at $1000 \mathrm{rpm}$ for 5 minutes, the cells were resuspended in PBS containing $2 \%$ BSA. Suspended AT cells were subjected to flow cytometric analysis.

Aliquots of the cell suspension were stained with primary mAbs directed against the extracellular domain of KDR (Sigma) and CD31 (BD Pharmingen, San Diego, California). After washing the cells with PBS twice, they were incubated with anti-mouse IgG $A b$ conjugated with FITC as the secondary Ab. Stained cells were fixed with $1 \%$ paraformaldehyde in PBS $\mathrm{pH}$ 7.5) and immediately analyzed by flow cytometry (Beckton-Dickinson, Franklin Lakes, New Jersey).

\section{Measurement of VEGF Protein in the Cell Culture Medium}

VEGF protein in cell-culture medium was measured using a commercially available VEGF sandwich immunoassay kit (R\&D Systems, Minneapolis, Minnesota) according to the manufacturer's instructions (Shintani et al, 2001a). The assay system has no cross-reactivity with platelet-derived growth factor (PDGF), basic fibroblast growth factor (bFGF), or epidermal growth factor (EGF). The coefficient of variation of interassay determinations was between 6.2 and $8.8 \%$

\section{Role of Autocrine VEGF in EPC Differentiation}

Human MNCs produce and secrete VEGF protein (Hojo et al, 2000), which could modulate the differentiation of PB-MNCs into EPCs through an autocrine mechanism (Risau, 1995). We examined the role of VEGF released from PB-MNCs on the differentiation of PB-MNCs into EPCs. For this purpose, we tested the effects of a neutralizing anti-VEGF mAb $(50,100$, and $500 \mathrm{ng} / \mathrm{mL}$, R\&D Systems) (Tsurumi et al, 1997), a nonspecific tyrosine kinase inhibitor genistein (100 $\mu \mathrm{mol} / \mathrm{L}$, Sigma), and a specific KDR-receptor tyrosine kinase inhibitor SU1498 (1 and $10 \mu \mathrm{mol} / \mathrm{L}$, Calbiochem, San Diego, California) (Shen et al, 1999) on the differentiation of PB-MNCs into EPCs. The effects of the $\mathrm{mAb}$ and the tyrosine kinase inhibitors on the number of cell clusters and of EPC-like AT cells were examined. The effect of mouse lgG $(500 \mathrm{ng} / \mathrm{mL}$, Sigma) on EPC differentiation was also evaluated as a control for the anti-VEGF mAb-treatment.

\section{EPC Migration Assay}

EPC migration was analyzed using a modified Boyden chamber apparatus (Neuroprobe, Gaithersburg, Maryland) (Murohara et al, 1999). Polyvinylpyrrolidone (PVP)-free polycarbonate filters with multiple pores (8 $\mu \mathrm{m}$ in diameter) were coated with $0.1 \%$ gelatin and 50 $\mu \mathrm{g} / \mathrm{mL}$ fibronectin and were dried under air for 6 hours. Culture medium $(25 \mu \mathrm{L})$ supplemented with $1 \%$ FBS and rhVEGF (10 ng/mL) was placed in the lower chamber of the apparatus. EPCs were isolated from culture plates from the normoxic and hypoxic groups. After a coated PVP-free polycarbonate filter was placed between the lower and upper chambers, $5 \times$ $10^{5}$ EPCs suspended in $50 \mu \mathrm{L}$ of Medium-199 containing 1\% FBS without VEGF were seeded in the upper chamber. The whole apparatus was then incubated for 5 hours at $37^{\circ} \mathrm{C}$ in a humidified incubator with $95 \%$ air and $5 \% \mathrm{CO}_{2}$ to render EPCs able to migrate through the membrane. After the incubation period, the filter was removed, and nonmigrated EPCs on the upper side of the filter were scraped-off with a rubber. The filters were then fixed with methanol for 10 minutes and stained with Giemsa solution. Migrated EPCs attached to the lower side of the filter were counted in three randomly selected microscopic fields $(\times 100)$ in each chamber, and the average number of migrated cells were calculated. All experiments were performed in triplicate, and data were expressed as the number of migrated cells/field.

\section{In Vivo Angiogenesis Model: Induction of Neovascularization by EPC Transplantation in a Rat Model of Unilateral Hindlimb Ischemia}

In a previous study, we showed that transplanted human EPCs participated in postnatal neovascularization in a rat model of surgically induced hindlimb ischemia. In addition, transplantation of EPCs augmented ischemia-induced neovascularization (Murohara et al, 2000). In the present study, we first examined whether transplanted EPCs participated in capillary formation in the ischemic hindlimb tissue of immunodeficient nude rats. For transplantation of human EPCs, immunodeficient (F344/N rnu/rnu) nude rats (Clea Japan, Pennington, New Jersey) were used to avoid graft-versus-host reactions. Male nude rats ( $n$ $=8$ ) were anesthetized with an sodium pentobarbital (50 mg/kg, ip), after which the left femoral artery and vein were excised to induce limb ischemia. Rats then received fluorescence-labeled (PKH2-GL, Sigma) EPCs $\left(3 \times 10^{5}\right.$ cells/rat) intramuscularly at the ischemic thigh skeletal muscles using a 26 -gauge needle. Two weeks after the surgery and cell transplantation, animals were killed with overdose of pentobarbital. Ischemic skeletal muscle samples were isolated, embedded, and snap-frozen in OCT compound (TissueTek, Bayer Corporation, New Haven, Connecticut). Six micrometer multiple frozen sections were prepared from each specimen so that the muscle fibers were oriented in a transverse fashion. Fluorescence microscopy was used to detect transplanted EPCs with fluorescence incorporated into the ischemic tissue.

We next examined whether hypoxia-conditioned EPCs elicit a greater angiogenic efficiency as compared with normoxia-conditioned EPCs when they were transplanted into ischemic tissues. Male nude rats $(n=17)$ were subjected to unilateral hindlimb ischemia by resecting the left femoral artery and vein under anesthesia with sodium pentobarbital $(50 \mathrm{mg} /$ 
$\mathrm{kg}$, ip). On Day 3 of hindlimb ischemia, rats were injected with either normoxia-conditioned EPCs $(n=$ $5)$, hypoxia-conditioned EPCs $(n=5)\left(2 \times 10^{5}\right.$ cells/ animal), or saline (as a control, $n=7$ ) in the ischemic thigh adductor muscle area. Regional hindlimb blood flow was evaluated by LDBF and compared among the three groups for the subsequent 3 weeks as mentioned below. Animal experiments were approved by the Institutional Animal Care and Use Committee.

\section{Laser Doppler Blood Flowmetry}

We measured the ratio of the ischemic (left)/normal (right) hindlimb blood flow using an LDBF analyzer (Moor Instruments, Devon, United Kingdom) (Murohara et al, 1998a). Low to no flow is displayed as dark blue, whereas high flow is displayed as red to white. Before initiating laser scanning, rats were placed for 15 minutes on a heating plate kept at $37^{\circ} \mathrm{C}$ to minimize body temperature changes. After scanning them, the stored images were subjected to computerassisted quantification of blood flow, and the average flow of the ischemic and nonischemic feet was calculated. The LDBF value was then expressed as the ratio of left (ischemic) to right (nonischemic) limb blood flow to avoid data variations caused by ambient light and room temperature. The ischemic/normal LDBF ratio was compared among the 3 groups.

\section{Statistics}

Data are expressed as mean $\pm \mathrm{SE}$. Differences between the normoxic and hypoxic groups were analyzed by paired Student's $t$ test. In a series of in vivo animal experiments, differences among the three groups were analyzed by one-way ANOVA followed by Scheffe's comparison between any two groups. Differences with a $p<0.05$ were considered statistically significant.

\section{Acknowledgments}

We thank K. Kimura and K. Moriyama for technical assistance.

\section{References}

Asahara T, Masuda H, Takahashi T, Kalka C, Pastore C, Silver M, Kearne M, and Isner JM (1999). Bone marrow origin of endothelial progenitor cells responsible for postnatal vasculogenesis in physiological and pathological neovascularization. Circ Res 85:221-228.

Asahara T, Murohara T, Sullivan A, Silver M, van der Zee R, Li T, Witzenbichler B, Schatteman G, and Isner JM (1997). Isolation of putative progenitor endothelial cells for angiogenesis. Science 275:964-967.

Brogi E, Schatteman G, Wu T, Kim EA, Varticovski L, Keyt B, and Isner JM (1996). Hypoxia-induced paracrine regulation of VEGF receptor expression. J Clin Invest 97:469-476.

Carmeliet P (2000). Mechanisms of angiogenesis and arteriogenesis. Nat Med 6:389-395.

DeLisser HM, Christofidou-Solomidou M, Strieter RM, Burdick MD, Robinson CS, Wexler RS, Kerr JS, Garlanda C,
Merwin JR, Madri JA, and Albelda SM (1997). Involvement of endothelial PECAM-1/CD31 in angiogenesis. Am J Pathol 151:671-677.

Folkman J (1995). Angiogenesis in cancer, vascular, rheumatoid and other disease. Nat Med 1:27-30.

Garlanda C and Dejana E (1997). Heterogeneity of endothelial cells: Specific markers. Arterioscler Thromb Vasc Biol 17: 1193-1202.

Gunsilius E, Duba HC, Petzer AL, Kahler CM, Grunewald K, Stockhammer G, Gabl C, Dirnhofer S, Clausen J, and Gastl G (2000). Evidence from a leukemia model for maintenance of vascular endothelium by bone-marrow-derived endothelial cells. Lancet 355:1688-1691.

Hojo Y, Ikeda U, Zhu Y, Okada M, Ueno S, Arakawa H, Fujikawa H, Katsuki T, and Shimada K (2000). Expression of vascular endothelial growth factor in patients with acute myocardial infarction. J Am Coll Cardiol 35:968-973.

Isner JM and Asahara T (1999). Angiogenesis and vasculogenesis as therapeutic strategies for postnatal neovascularization. J Clin Invest 103:1231-1236.

Kalka C, Masuda H, Takahashi T, Kalka-Moll WM, Silver M, Kearney M, Li T, Isner JM, and Asahara T (2000). Transplantation of ex vivo expanded endothelial progenitor cells for therapeutic neovascularization. Proc Natl Acad Sci USA 97:3422-3427.

Kanno S, Oda N, Abe M, Terai Y, Ito M, Shitara K, Tabayashi K, Shibuya M, and Sato $Y$ (2000). Roles of two VEGF receptors, Flt- 1 and KDR, in the signal transduction of VEGF effects in human vascular endothelial cells. Oncogene 19: 2138-2146.

Leung DW, Cachianes G, Kuang WJ, Goeddel DV, and Ferrara N (1989). Vascular endothelial growth factor is a secreted angiogenic mitogen. Science 246:1306-1309.

Lin Y, Weisdorf DJ, Solovey A, and Hebbel RP (2000). Origins of circulating endothelial cells and endothelial outgrowth from blood. J Clin Invest 105:71-77.

Millauer B, Wizigmann-Voos S, Schnurch $\mathrm{H}$, Martinez $\mathrm{R}$, Moller NP, Risau W, and Ullrich A (1993). High affinity VEGF binding and developmental expression suggest Flk-1 as a major regulator of vasculogenesis and angiogenesis. Cell 72:835-846.

Murohara T, Asahara T, Silver M, Bauters C, Masuda H, Kalka C, Kearney M, Chen D, Symes JF, Fishman MC, Huang PL, and Isner JM (1998a). Nitric oxide synthase modulates angiogenesis in response to tissue ischemia. $\mathrm{J}$ Clin Invest 101:2567-2578.

Murohara T, Horowitz JR, Silver M, Tsurumi Y, Chen D, Sullivan A, and Isner JM (1998b). Vascular endothelial growth factor/vascular permeability factor enhances vascular permeability via nitric oxide and prostacyclin. Circulation 97:99107.

Murohara T, Ikeda H, Duan J, Shintani S, Sasaki Ki, Eguchi H, Onitsuka I, Matsui K, and Imaizumi T (2000). Transplanted cord blood-derived endothelial precursor cells augment postnatal neovascularization. J Clin Invest 105:1527-1536.

Murohara T, Witzenbichler B, Spyridopoulos I, Asahara T, Ding B, Sullivan A, Losordo DW, and Isner JM (1999). Role of endothelial nitric oxide synthase in endothelial cell migration. Arterioscler Thromb Vasc Biol 19:1156-1161. 
Namiki A, Brogi E, Kearney M, Kim EA, Wu T, Couffinhal T, Varticovski L, and Isner JM (1995). Hypoxia induces vascular endothelial growth factor in cultured human endothelial cells. J Biol Chem 270:31189-31195.

Risau W (1995). Differentiation of endothelium. FASEB J 9:926-933.

Risau W (1997). Mechanisms of angiogenesis. Nature 386: 671-674.

Shen BQ, Lee DY, Gerber HP, Keyt BA, Ferrara N, and Zioncheck TF (1998). Homologous up-regulation of KDR/ Flk-1 receptor expression by vascular endothelial growth factor in vitro. J Biol Chem 273:29979-29985.

Shen BQ, Lee DY, and Zioncheck TF (1999). Vascular endothelial growth factor governs endothelial nitric-oxide synthase expression via a KDR/FIk-1 receptor and a protein kinase C signaling pathway. J Biol Chem 274:33057-33063.

Shintani S, Murohara T, Ikeda H, Ueno T, Honma T, Katoh A, Sasaki K, Shimada T, Oike Y, and Imaizumi T (2001a). Mobilization of endothelial progenitor cells in patients with acute myocardial infarction. Circulation 103:2776-2779.

Shintani S, Murohara T, Ikeda H, Ueno T, Sasaki K, Duan J, and Imaizumi T (2001b). Augmentation of postnatal neovascularization with autologous bone marrow transplantation. Circulation 103:897-903.

Shweiki D, Itin A, Soffer D, and Keshet E (1992). Vascular endothelial growth factor induced by hypoxia may mediate hypoxia-initiated angiogenesis. Nature 359:843-845.
Takahashi T, Kalka C, Masuda H, Chen D, Silver M, Kearney M, Magner M, Isner JM, and Asahara T (1999). Ischemia- and cytokine-induced mobilization of bone marrow-derived endothelial progenitor cells for neovascularization. Nat Med 5:434-438.

Tateishi-Yuyama E, Matsubara $\mathrm{H}$, Murohara $T$, Ikeda $\mathrm{U}$, Shintani S, Masaki H, Amano K, Kishimoto Y, Yoshimoto K, Akashi H, Shimada K, Iwasaka T, and Imaizumi T (2002). Therapeutic angiogenesis for patients with limb ischaemia by autologous transplantation of bone-marrow cells: A pilot study and a randomised controlled trial. Lancet 360:427435 .

Tsurumi Y, Murohara T, Krasinski K, Chen D, Witzenbichler B, Kearney M, Couffinhal T, and Isner JM (1997). Reciprocal relation between VEGF and NO in the regulation of endothelial integrity. Nat Med 3:879-886.

Vasa M, Fichtlscherer S, Adler K, Aicher A, Martin H, Zeiher $A M$, and Dimmeler $S$ (2001). Increase in circulating endothelial progenitor cells by statin therapy in patients with stable coronary artery disease. Circulation 103:2885-2890.

Yamaguchi TP, Dumont DJ, Conlon RA, Breitman ML, and Rossant J (1993). Flk-1, and flt-related receptor tyrosine kinase is an early marker for endothelial cell precursors. Development 118:489-498. 